\title{
Development of SPR biosensor for simultaneous detection of multiplex respiratory viruses ${ }^{1}$
}

\author{
Lei Shi ${ }^{\mathrm{a}, \mathrm{b}}$, Qiuxiang Sun ${ }^{\mathrm{c}}$, Jian'an $\mathrm{He}^{\mathrm{d}}$, Hua Xu ${ }^{\mathrm{d}}$, Chunxiao Liu ${ }^{\mathrm{d}}$, Chunzhong Zhao ${ }^{\mathrm{d}}$, Yunqing \\ $\mathrm{Xu}^{\mathrm{d}}$, Changlin Wu $\mathrm{W}^{\mathrm{d}}$, Junjian Xiang ${ }^{\mathrm{a}}$, Dayong Gu${ }^{\mathrm{d}}$, Jun Long ${ }^{\mathrm{e}}$ and Hekui Lan ${ }^{\mathrm{e}, *}$ \\ ${ }^{a}$ Guangdong Province Key Laboratory of Molecular Immunology and Antibody Engineering, Antibody \\ Engineering Center of Jinan University, Guangzhou, 510632, P.R. China \\ ${ }^{b}$ Shenzhen Academy of Inspection and Quarantine, Shenzhen, 518010, P.R. China \\ ${ }^{c}$ Feng Gang Hospital, Dongguan, 523695, P.R. China \\ ${ }^{d}$ Central Laboratory of Health Quarantine, Shenzhen International Travel Health Care Center, \\ Shenzhen Entry-exit Inspection and Quarantine Bureau, Shenzhen, 518033, P.R. China \\ ${ }^{e}$ Department of Pediatrics, Zhujiang Hospital, Southern Medical University, Guangzhou, 510282, P.R. \\ China
}

\begin{abstract}
A surface plasmon resonance (SPR)-based biosensor was developed for specific detection of nine common respiratory virus, including influenza $A$ and influenza $B, H 1 N 1$, respiratory syncytial virus (RSV), parainfluenza virus 1-3 (PIV1, 2, 3), adenovirus, and severe acute respiratory syndrome coronavirus (SARS). The SPR biosensor was developed by immobilizing nine respiratory virus-specific oligonucleotides in an SPR chip. To increase the biosensor sensitivity, biotin was used to label the PCR primer and further amplify the signal by introducing streptavidin after hybridization. Throat swab specimens representing nine common respiratory viruses were tested by the innovative SPR-based biosensor to evaluate the sensitivity, specificity and reproducibility of this method. Results suggest that this biosensor has the potential to simultaneously identify common respiratory viruses.
\end{abstract}

Keywords: SPR, respiratory virus, high throughput, label-free, gene chip

\section{Introduction}

Respiratory viruses are the primary cause of acute respiratory diseases in both the upper and lower respiratory tracts. Many kinds of respiratory viruses have been identified, including influenza A and influenza B, H1N1, respiratory syncytial virus (RSV), parainfluenza virus (PIV1, 2, 3), adenovirus, and severe acute respiratory syndrome coronavirus (SARS). Effective and rapid diagnosis can contribute to the control of disease outbreaks. Rapid diagnosis can confirm clinical suspicion of

\footnotetext{
${ }^{1}$ Lei Shi and Qiuxiang Sun contributed equally to this work.

* Address for correspondence: Hekui Lan, Department of Pediatrics, Zhujiang Hospital, Southern Medical University, Guangzhou, 510282, P.R. China. Tel.: 086-020-62782999; Fax: 086-0755-83394162; E-mail: hk-lan@163.com.
}

0959-2989/15/\$35.00 @ 2015 - IOS Press and the authors. 
infection and guide further treatment and prognosis. These outcomes help to mitigate healthcare costs and allow early public health intervention to prevent the spread of respiratory viruses. Therefore, a rapid method for early detection of respiratory viruses is urgently required to enable doctors to ascertain the specific virus quickly and to prescribe an appropriate course of treatment [1]. Current methods to diagnose respiratory viral infection utilize several different formats, including direct virus detection via isolation of the virus in a cell culture, or identification of viral nucleic acid or antigen, and serological tests. Direct virus detection is not suitable for the routine diagnosis of respiratory viral infection because the procedures require considerable skilled expertise and are often time-consuming. Serological approaches include nucleic acid sequence-based amplification (NASBA) [2], Restriction endonuclease analysis (REA) [3, 4] and direct immunofluorescent assays [5]. Enzyme-linked immunosorbent assays (ELISAs) are commercially available [6,7], but they usually require long assay times and a labor-intensive sampling process. Recently, testing of ribonucleic acid (RNA) viral cultures using reverse transcription-polymerase chain reactions (RT-PCR) has also become common, whereby viral nucleic acids extracted from samples are transcribed into complementary deoxyribonucleic acid (cDNA) which is then amplified and analyzed by fluorescence or luminescence $[8,9]$. Despite the simplicity of this method, it cannot provide specific sequence information and produces higher false positive results arising from the amplification of non-specific sequences.

In light these limitations, new methodologies and devices aimed at respiratory virus diagnosis have been reported in recent decades. Among all of these technologies, surface plasmon resonance (SPR) exhibits potential as a sensitive optical biosensor for real-time and label-free detection [10-13]. The goal of this study is to overcome the inherent disadvantages of a traditional PCR-based method and implement rapid and high-throughput identification of respiratory viruses. We combined the advantages of SPR and the use of a gene chip to simultaneously detect the genes of respiratory viruses in PCR products. A single-strand amine group is used to label oligonucleotide probes of nine respiratory viruses and immobilized in parallel on onto self-assembled monolayers (SAM) on the surface of a12-mercaptododecanoic acid (MDA) via amine chemistry [14]. This method is extremely efficient in terms of high throughput for respiratory virus DNA detection. The respiratory virus target gene was extracted from bacterial culture samples and amplified by PCR technology; PCR products were then analyzed by SPR technology. The basic principle of the proposed method is illustrated in Scheme 1 . The sensitivity, specificity and reproducibility of this method were also evaluated in detail.

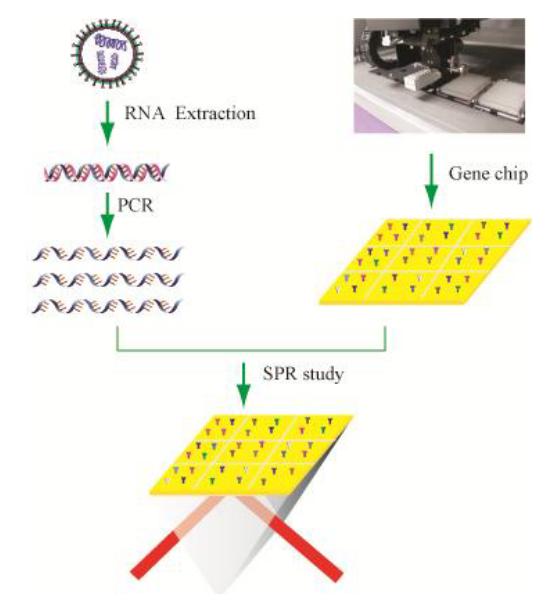

Scheme. 1. Schematic illustration of the strategy for the detection of respiratory viruses. The producer included RNA extraction from throat swab specimens, amplification vi one-step RT-PCR, gene chip fabrication and analysis by SPR. 


\section{Materials and methods}

\subsection{Instrument and reagents}

A PlexArray ${ }^{\mathrm{TM}}$ HT system (Plexera ${ }^{\mathrm{R}}$ Bioscience, USA) was used for this study. A C1000 PCR thermal cycler and a Bio-Rad gel imager were obtained from Bio-Rad Laboratories, Inc. A Nano Drop 1000 spectrophotometer was obtained from Thermo Fisher Scientific, Inc. MDA was purchased from Sigma, and 2-aminoethanol hydrochloride was obtained from Shanghai Ruji Technology Co., Ltd. 1Ethyl-3-(3-dimethylaminopropyl) carbodiimide (EDC) and N-hydroxysuccinimide (NHS) were obtained from Shanghai Li Zhu Dong Feng Biotechnology Co. Streptavidin (SA) was obtained from Beijing Biosynthesis Biotechnology Co., LTD. All other chemicals were analytical grade reagents. All biological reagents were stored at $4{ }^{\circ} \mathrm{C}$, and all solutions were prepared with ultrapure water $(>18.2$ $\mathrm{M} \Omega \mathrm{cm}^{-1}$ ). A $0.01 \mathrm{~mol} \mathrm{~L}^{-1}$ phosphate-buffered saline solution (PBS, $\mathrm{pH}$ 7.4) was prepared by dissolving $0.2 \mathrm{~g} \mathrm{KCl}, 8.0 \mathrm{~g} \mathrm{NaCl}, 0.24 \mathrm{~g} \mathrm{KH}_{2} \mathrm{PO}_{4}$ and $1.44 \mathrm{~g} \mathrm{Na}_{2} \mathrm{HPO}_{4}$, in $1000 \mathrm{~mL}$ water.

Oligonucleotide probes labeled with synthetic amino $\left(\mathrm{NH}_{2}\right)$ groups and biotin-labeled primers were obtained from Invitrogen (USA). The sequences of primers and probes used for these experiments are listed in Table 1.

\subsection{SPR biochip fabrication and surface modification}

Thin gold chips were prepared by vacuum evaporation and of $2.5 \mathrm{~nm}$ chromium and $47 \mathrm{~nm}$ gold onto SF10 glass wafers. The gold chip surface was then cleaned by oxygen plasma treatment for a period of one minute. The chips were then immersed in $1 \mathrm{mM}$ MDA for 16 hours. After the formation of MDA SAMs, the chips were thoroughly rinsed with ethanol and then dried in a stream of nitrogen.

\subsection{Gene chip design}

Table 1

Sequence of primers and probes

\begin{tabular}{|c|c|c|}
\hline Name & Primes (5'-3') (F/R) & Probe (5'-3') \\
\hline Influ A & $\begin{array}{l}\text { AAAGCGAATTTCAGTGTGAT; } \\
\text { GAAGGCAATGGTGAGATTT }\end{array}$ & AGGGCTTTCACCGAAGAGGG \\
\hline Influ B & $\begin{array}{l}\text { GTCCATCAAGCTCCAGTTTT } \\
\text { TCTTCTTACAGCTTGCTTGC }\end{array}$ & AACGAAGTAGGTGGAGACGGAGG \\
\hline PIV1 & $\begin{array}{l}\text { ACCTACAAGGCAACAACATC } \\
\text { CTTCCTGCTGGTGTGTTAAT }\end{array}$ & CAAACGATGGCTGAAAAAGGGA \\
\hline PIV2 & $\begin{array}{l}\text { CCATTTACCTAAGTGATGGAA } \\
\text { CGTGGCATAATCTTCTTTTT }\end{array}$ & AATCCGCAAAAGCTGTTCAGTCAC \\
\hline PIV3 & $\begin{array}{l}\text { GGAGCATTGTGTCATCTGTC; } \\
\text { TAGTGTGTAATGCAGCTCGT }\end{array}$ & ACCCAGTCATAACTTACTCAACAGCAAC \\
\hline RSV & $\begin{array}{l}\text { TTTCCACAATATTAAGTGTCAA } \\
\text { TCATCCCATACTTTTCTGTTA }\end{array}$ & CCATGTGAATTCCCTGCATCAAT \\
\hline SARS & $\begin{array}{l}\text { CAGAACGCTG TAGCTTCAAAAATCT } \\
\text { TCAGAACCCTGTGATGAATCAACAG }\end{array}$ & TCTGCGTAGGCAATCC \\
\hline $\mathrm{ADV}$ & $\begin{array}{l}\text { CAGTGGTCTTACATGCACATC } \\
\text { CAGCACGCCGCGGATGTCAAAGT }\end{array}$ & CACCGAGACGTACTTCAGCCTG \\
\hline H1N1 & $\begin{array}{l}\text { TTGATGATGGTTTCCTGGACA } \\
\text { ACTTTCCATGCACGTGTTATCGC }\end{array}$ & GGACTACCACGATTCAAATGTG \\
\hline
\end{tabular}


To activate the carboxyl groups before printing, the sensor chip was immersed into an aqueous mixture of $0.4 \mathrm{M}$ EDC and $0.1 \mathrm{NHS}$, a $10 \mu \mathrm{M}$ solution of specific respiratory virus probes in PBS (10 $\mathrm{mM}, \mathrm{pH}$ 7.4) were printed onto the activated surface by an automated Genetix QArray mini printer and then incubated at room temperature for 2 hours. After incubation, the sensor chip was washed with milliQ water, and then treated with $1 \mathrm{mM}$ ethanolamine $(\mathrm{pH}=8.5)$ blocking solution at room temperature for a further 10 minutes in order to deactivate the remaining NHS carbonate ester groups.

\subsection{PCR amplification}

The respiratory virus RNA from each $200 \mu \mathrm{L}$ throat swab specimen was extracted using EZ1 Advanced Instruments (QIAGEN, German) according to manufacturer's protocol. The target RNA amplification was performed by one-step PCR. Biotin-labeled primers were used for RT-PCR. The RT-PCR assay protocol is as follows:

A $0.3 \mu \mathrm{L}$ template RNA was added to a mixture containing $3 \mu \mathrm{L}$ of $10 \times$ PCR buffer (TAKARA), 9.6 $\mu \mathrm{L}$ of a primer mixture, $0.5 \mu \mathrm{L}$ of Taq DNA polymerase, $0.25 \mu \mathrm{L}$ of AMV RT, and $0.6 \mu \mathrm{L}$ of a dNTP mixture; sterile water was then added to reach a total volume of $30 \mu \mathrm{L}$. Tubes without DNA templates were included in each experiment as blank control samples. Samples were amplified in a thermocycler under the following conditions: initial PCR activation at $42^{\circ} \mathrm{C}$ for 40 minutes, then at $95^{\circ} \mathrm{C}$ for two minutes. Two-step cycling was conducted at $95^{\circ} \mathrm{C}$ for 30 seconds, then at $54^{\circ} \mathrm{C}$ for 30 seconds, and $72^{\circ} \mathrm{C}$ for 30 seconds; five cycles were performed. Three-step cycling was conducted at $95^{\circ} \mathrm{C}$ for 30 seconds, $49^{\circ} \mathrm{C}$ for 30 seconds, and then at $72^{\circ} \mathrm{C}$ for 30 seconds; 30 cycles were performed. Final extension was conducted at $72^{\circ} \mathrm{C}$ for two minutes

DNA concentration was determined by an ND 1000 spectrophotometer (Nano Drop Technology, $\mathrm{DE}$ ), and the extracts were stored at $-20^{\circ} \mathrm{C}$. PCR products were denatured by a 5 -minute incubation at $95^{\circ} \mathrm{C}$ followed by cooling on ice for 1 minute. Screening of PCR products was performed by gel electrophoresis on $2 \%$ agarose gel, stained with ethidium bromide, and visualized using a UV transilluminator and compared to a DNA ladder (Ready-to-Use ${ }^{\mathrm{TM}}$ Plus 100 bp DNA Ladder).

\subsection{SPR study}

SPR experiments were performed by a PlexArray ${ }^{\mathrm{TM}} \mathrm{HT}$ system (Plexera Bioscience, American). The SPR chip modified with oligonucleotide probes was docked into the SPR instrument. Phosphate buffered saline (PBS) solution (10 mM, pH 7.4) was used as the running buffer with a flow rate of 50 $\mu \mathrm{L} \min ^{-1}$ with a peristaltic pump to obtain a stable baseline. The hybridization measurement was conducted by injecting $10 \mu \mathrm{M}$ of the target PCR product solution with a peristaltic pump, at a flow rate of $5 \mu \mathrm{L} \mathrm{min}{ }^{-1}$. The reaction was monitored for five minutes, and the sensor chip was automatically washed with hybridization buffer to remove the unbound DNA material. After hybridization, $60 \mu \mathrm{g}$ $\mathrm{mL}^{-1}$ of SA solution was pumped to a flow cell for five minutes in order to enhance the signal. Reported response unit $(\Delta \mathrm{RU})$, the analytical signal, was derived by the difference between the final reflectivity intensity and the reflectivity intensity recorded before hybridization.

After hybridization, the surface was regenerated with $100 \mathrm{mM}$ of $\mathrm{NaOH}$ solution to obtain a probe for reuse in a new cycle of hybridization reactions.

\section{Results}




\subsection{Gel electrophoresis of PCR products}

In this study, to enhance sensitivity for the detection of respiratory viruses, the target RNA was amplified by RT-PCR technology. Figure 1 illustrates the results of agarose gel electrophoresis, which verify that specific target segments for nine respiratory viruses were successfully amplified from extracted RNA.

As an extension of the practical use of PCR, multiplex PCR has the potential to amplify more than one target sequence by using multiple primers in a reaction mixture without compromising the utility of the experiment $[15,16]$. Thus, it is an essential cost-saving technique for large scale clinical application. For our study, we conducted multiplex PCR to amplify the target RNA. A multiplex PCR for the simultaneous amplification of four viral genes (ADV, RSV, PIV2 and SARS) was validated by agarose gel electrophoresis, as shown in Figure 2. This approach has been successfully applied to the amplification of two kinds of viral gene mixtures (influenza B and ADV).

\subsection{Measurement specificity}

All PCR products were diluted with PBS $(10 \mathrm{mM}, \mathrm{pH} 7.4)$ up to a desired concentration. The analysis of PCR product results were conducted by SPR technology. To allow hybridization with the immobilized probe, PCR products consisting of double-stranded DNA fragments require a

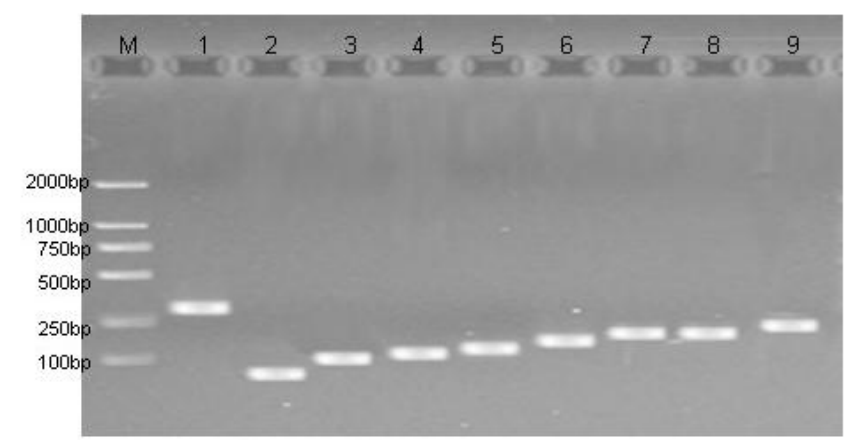

Fig. 1. Gel electrophoresis of PCR products from nine respiratory virus DNA templates. Lane M: DL2000. (1) ADV (297 bp); (2) SARS (68 bp); (3) influenza A (104 bp); (4) PIV2 (116 bp); (5) PIV1 (129 bp); (6) influenza B (145 bp); (7) RSV (155 bp); (8) PIV3 (154 bp; and (9) H1N1 (202bp).
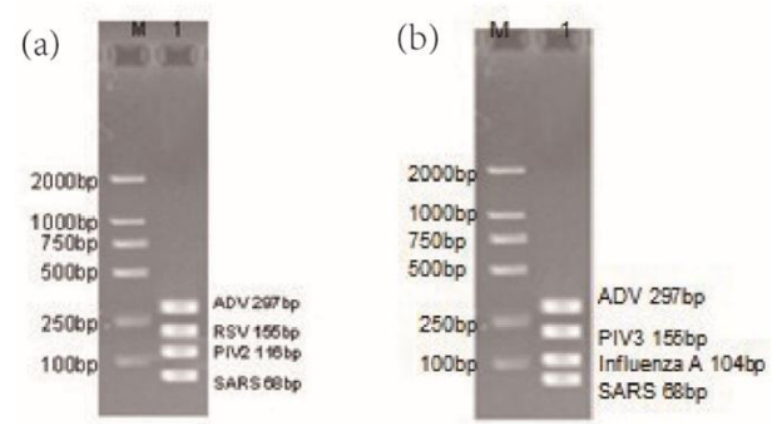

Fig. 2. Gel electrophoresis of multiplex PCR products. (a) Lane M: DL2000. Lane 1 (from top to bottom): ADV (297 bp), RSV (155 bp), PIV2 (116 bp), SARS (68 bp); (b) Lane M: DL2000; Lane 1 (from top to bottom): ADV (297 bp), PIV3 (154 bp), influenza A (104 bp), and SARS (68 bp). 
denaturation step by employing a 10 minute thermal treatment prior to injection. Typical response curves of respiratory virus detection are shown in Figure 3. Different PCR products demonstrated specific responses to complementary DNA probes. No cross-hybridization occurred between the PCR products and probes which were not fully complementary. Furthermore, the SPR signal can be enhanced by an injection of SA solution. Unfortunately, probes which were not fully complementary to the PCR product produced small SPR signals. We postulate that this phenomenon is a result of nonspecific protein adsorption to the SAM of the MDA surface. These non-specific adsorptions can be minimized by using ethylene glycol alkane thiol self-assembled monolayers (PEG SAM) [17], oligo (ethylene glycol) methacrylate (OEGMA) based polymer brushes [18] or a polyrotaxane matrix [19]. All hybridized DNA must be removed before the gene chip can be reused. In this study, we used a $\mathrm{NaOH}(100 \mathrm{mM})$ solution as the regeneration reagent. Figure 3 illustrates that the baseline is near original values when the chip is regenerated with $\mathrm{NaOH}$ solution, indicating that hybridized DNA can be removed. SPR results confirm that the gene chip combined with the SPR technique provides a highly specific platform for high throughput detection of respiratory virus PCR products.
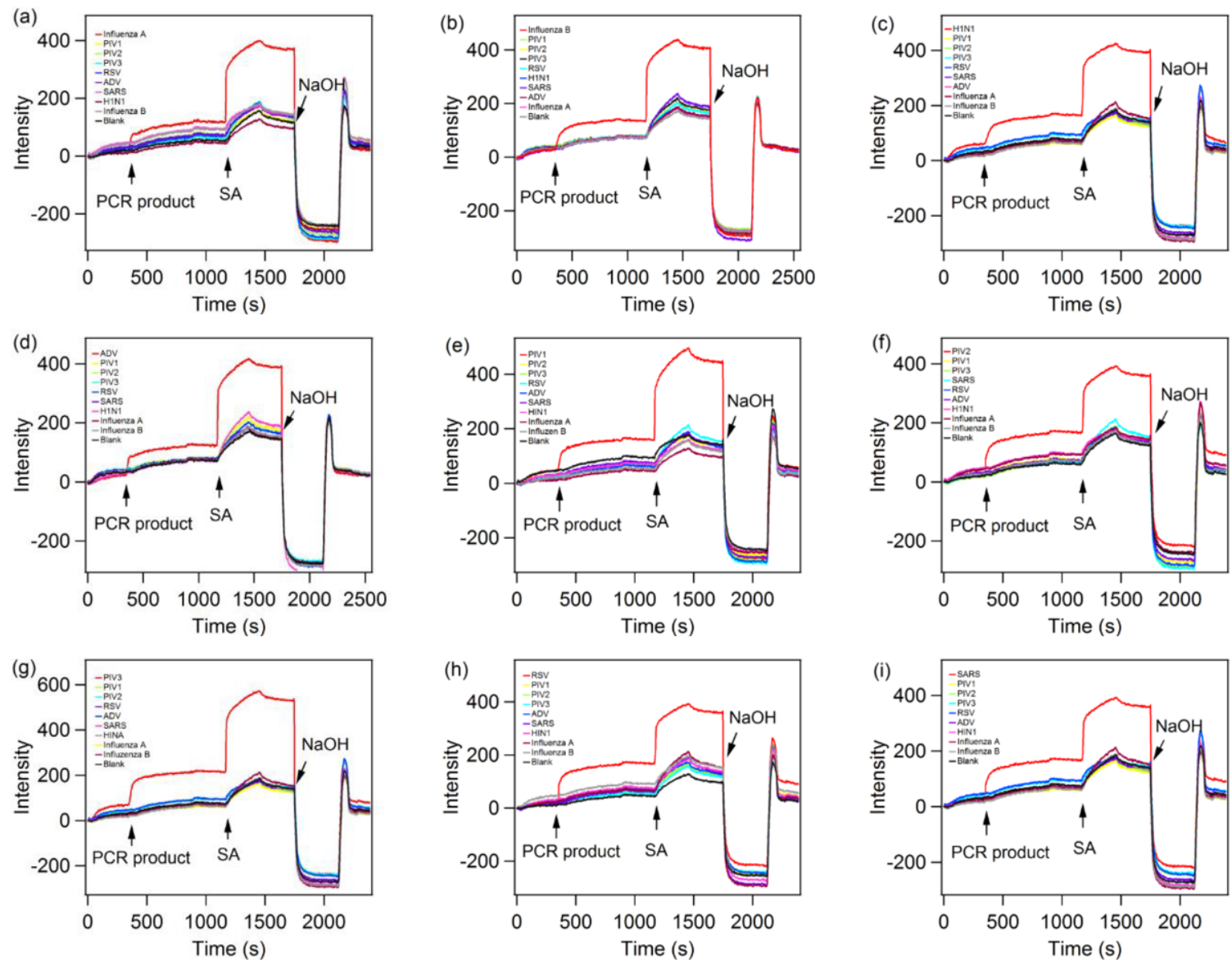

Fig. 3. Typical response curves for detection of nine respiratory virus PCR products: (a) influenza A; (b) influenza B; (c) H1N1; (d) ADV; (e) PIV1; (f) PIV2; (g) PIV3; (h) RSV; and (i) SARS. The standard procedure consists of running with PBS to obtain a baseline; PCR product was injected to display the specific hybridization. After hybridization, SA solution was used for signal enhancement. Finally, the SPR gene chip was regenerated with $100 \mathrm{mM}$ of $\mathrm{NaOH}$ solution. 

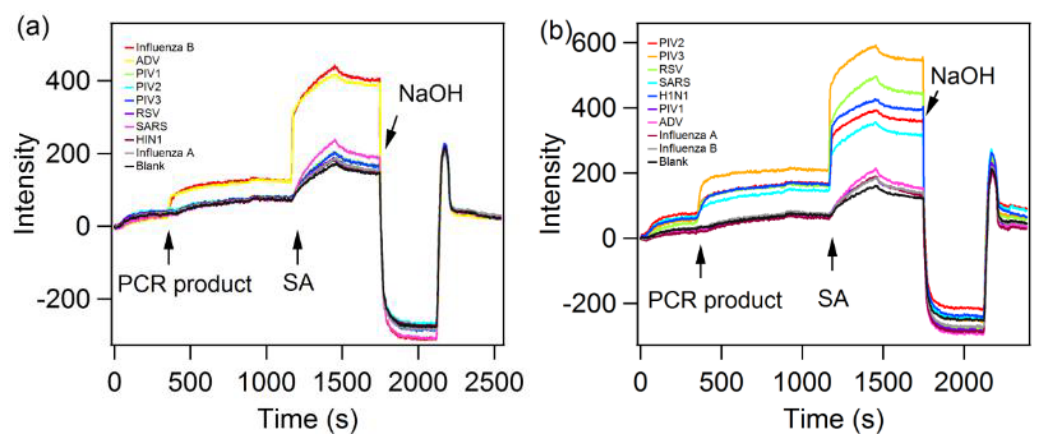

Fig. 4. Typical curves for detecting multiple PCR products. (a) Influenza B and an ADV mixture; (b) a PIV2, PIV3, RSV, SARS and H1N1 mixture.

PCR blanks containing all PCR mixture reagents (except the template DNA) were tested to identify any non-specific effects. Results confirmed that injection of control solutions obtained from PCR and conducted without a DNA template produced signal shifts similar to instrument noise.

In this study, we used a gene chip combined with SPR technology to analyze the multiplex PCR products. The main benefits of simultaneous detection of multiplex respiratory viruses in one PCR product are efficiency and a higher throughput. Two typical SPR sensor grams exhibiting multiplex PCR products detection are illustrated in Figure 4: the multiplex PCR of influenza B and ADV, and multiplex PCR of PIV2, PIV3, RSV, SARS and H1N1. Results verified that this platform enables us to simultaneously detect multiplex respiratory viruses with high specificity within minutes.

\subsection{Measurements repeatability}

In many cases, to reduce the difference between chips or reduce costs, the sensor chip needs to be reused; however, the repeatability of experiments must be estimated and DNA targets must be removed, but the probes must remain intact. A typical SPR response curve for four cycles of injection of influenza A PCR product onto specific probe spots and the removal of influenza A target DNA using $\mathrm{NaOH}$ are exhibited in Figure 5. Note that the SPR baseline returned to the original value after regeneration, indicating that the target can be completely removed by $\mathrm{NaOH}$ solution. Binding curves reconstructed from a typical SPR curve nearly overlap. An average signal obtained was 398 RU with a relative standard deviation was $0.7 \%$, indicating that the measurements exhibit good repeatability.

\subsection{Measurements sensitivity}

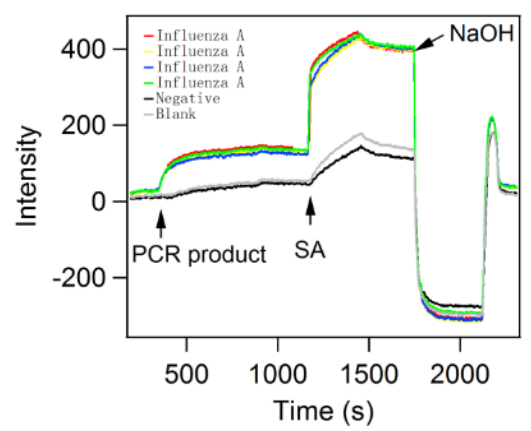

Fig. 5. Measurement repeatability tested by SPR using influenza A $(10 \mu \mathrm{M})$ as a model analyte. 

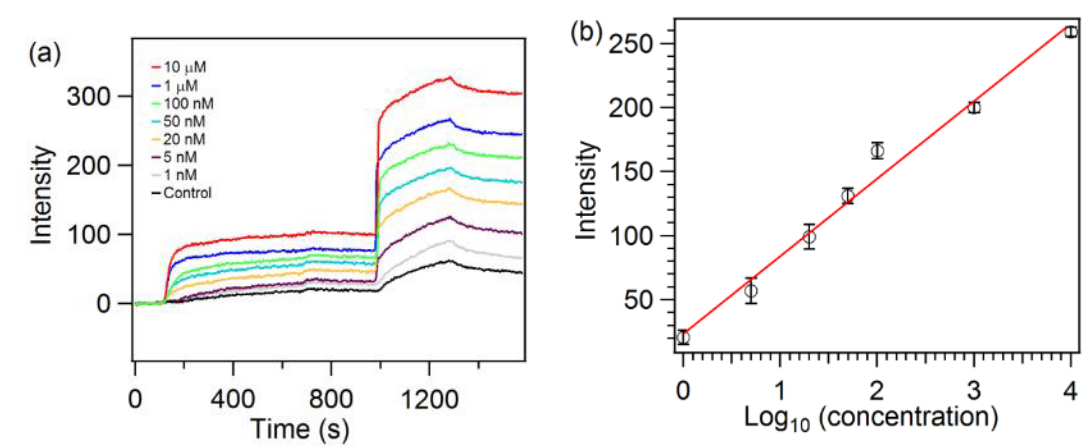

Fig. 6. SPR response to serial concentrations of influenza B PCR products. (a) Typical sensorgram showing the hybridization of serial concentrations of PCR products with influenza B probe; (b) dose-response analysis.

Table 2

The detection limit of nine respiratory viruses

\begin{tabular}{llllllllll}
\hline Virus & Influ A & InfluB & PIV $_{1}$ & PIV $_{2}$ & PIV $_{3}$ & RSV & ADV & SARA & H1N1 \\
LOD & $5 \mathrm{nM}$ & $1 \mathrm{nM}$ & $1 \mathrm{nM}$ & $2.5 \mathrm{nM}$ & $3.5 \mathrm{nM}$ & $3 \mathrm{nM}$ & $0.5 \mathrm{nM}$ & $2 \mathrm{nM}$ & $3 \mathrm{nM}$ \\
\hline
\end{tabular}

A serial concentration of PCR products was tested by SPR. Figure 6(a) confirms that SPR response signal values increased with increasing concentrations of PCR product of Influenza B. Based on collected SPR data, SPR signal values provide a linear correlation ranging from $1 \mathrm{nM}$ to $1 \mu \mathrm{M}$, as shown in Figure 6(b). With the detection limit of sensor response $\mathrm{S} / \mathrm{N}=3$, the lowest concentration of Influenza B that could be detected was $1 \mathrm{nM}$. The experimental limit of detection for nine kinds of virual PCR products are listed in Table 2.

\section{Discussion}

In this research, we have developed a gene chip SPR biosensor for the high throughput detection of nine different respiratory viral PCR products: influenza A and influenza B, H1N1, RSV, PIV1, 2, 3, adenovirus, and SARS). Our study confirms that the probes display specific hybridization properties which complement their target DNA in PCR products, and are amplified by single PCR and multiplex PCR. Multiplex PCR has been independently verified by agarose gel electrophoresis. To date, up to five combinations of multiplex PCR have been successfully amplified from the extracted RNA of respiratory viruses. The use of a high throughput gene chip combined with SPR facilitates that detection of multiplex PCR products selectively and simultaneously within 30 minutes. Furthermore, with biotin primer labeling, it is possible to enhance the limit of detection of this method with SA. Using this platform, we fabricated a SAM of MDA as the surface chemistry for probe immobilization because it is easy to prepare and use. However, we found that this surface exhibits a small amount of non-specific protein adsorption when it is combined with an SA solution. Further improvement to the matrix design to reduce the non-specific adsorption could be achieved. Specifically, the SPR biosensor combined with PCR amplification represents a new molecular tool for the high throughput and specific detection of respiratory viruses, which facilitates improvement in the efficacy of control and treatment of respiratory viral infections. 


\section{Acknowledgments}

This research is supported by the National Natural Science Foundation of China (No. 30972827 and No. 81171667), Shenzhen/Hong Kong Innovation Circle (No. ZYB200907090128A), Shenzhen Science and Technology Foundation (No. JCYJ20140419151618022), Shenzhen Science and Technology R \& D Foundation (No. ZYA201106080020A), Project supported by Shenzhen enter-exit inspection and quarantine bureau (No.SZ2014101), Guangdong Science and Technology Research Foundation (2012B031800126) and Shenzhen science and technology R \& D Foundation (No. ZYA201106080020A).

\section{References}

[1] R.L. Caygill, G.E. Blair and P.A. Millner, A review on viral biosensors to detect human pathogens, Analytica Chimica Acta 681 (2010), 8-15.

[2] T. Kievits, B. van Gemen, D. van Strijp, R. Schukkink, M. Dircks, H. Adriaanse, L. Malek, R. Sooknanan and P. Lens, NASBA isothermal enzymatic in vitro nucleic acid amplification optimized for the diagnosis of HIV-1 infection, Journal of Virological Methods 35 (1991), 273-286.

[3] V. Gouvea, C.H. Hoke, Jr. and B.L. Innis, Genotyping of hepatitis E virus in clinical specimens by restriction endonuclease analysis, Journal of Virological Methods 70 (1998), 71-78.

[4] K. Zierenberg, R. Raue and H. Muller, Rapid identification of "very virulent" strains of infectious bursal disease virus by reverse transcription-polymerase chain reaction combined with restriction enzyme analysis, Avian Pathology: Journal of the World Veterinary Poultry Association 30 (2001), 55-62.

[5] B. Rath, F. Tief, P. Obermeier, E. Tuerk, K. Karsch, S. Muehlhans, E. Adamou, S. Duwe and B. Schweiger, Early detection of influenza A and B infection in infants and children using conventional and fluorescence-based rapid testing, Journal of Clinical Virology: The Official Publication of the Pan American Society for Clinical Virology 55 (2012), 329-333.

[6] A.C. Boon, A.M. French, D.M. Fleming and M.C. Zambon, Detection of influenza a subtypes in community-based surveillance, Journal of Medical Virology 65 (2001), 163-170.

[7] C.H. Cho, M.K. Woo, J.Y. Kim, S. Cheong, C.K. Lee, S.A. An, C.S. Lim and W.J. Kim, Evaluation of five rapid diagnostic kits for influenza A/B virus, Journal of Virological Methods 187 (2013), 51-56.

[8] S.A. Butt, V.P. Maceira, M.E. McCallen and K.A. Stellrecht, Comparison of three commercial RT-PCR systems for the detection of respiratory viruses, Journal of Clinical Virology 61 (2014), 406-410.

[9] A. Piralla, C. Daleno, E. Pariani, P. Conaldi, S. Esposito, A. Zanetti and F. Baldanti, Virtual quantification of influenza A virus load by real-time RT-PCR, Journal of Clinical Virolog: the Official Publication of the Pan American Society for Clinical Virology 56 (2013), 65-68.

[10] W. Wang, M.W. Wolff, U. Reichl and K. Sundmacher, Model-based identification of adsorption kinetics from surface plasmon resonance experiments, Avian Pathology: Journal of the World Veterinary Poultry Association 1326 (2014), $125-129$.

[11] Y. Wang, Z. Ye, C. Si and Y. Ying, Monitoring of Escherichia coli O157:H7 in food samples using lectin based surface plasmon resonance biosensor, Food Chemistry 136 (2013), 1303-1308.

[12] D. Zhang, Y. Yan, Q. Li, T. Yu, W. Cheng, L. Wang, H. Ju and S. Ding, Label-free and high-sensitive detection of Salmonella using a surface plasmon resonance DNA-based biosensor, Journal of Biotechnology 160 (2012), $123-128$.

[13] H. Sipova and J. Homola, Surface plasmon resonance sensing of nucleic acids: a review, Analytica Chimica Acta 773 (2013), 9-23.

[14] L.A. Chrisey, G.U. Lee and C.E. O'Ferrall, Covalent attachment of synthetic DNA to self-assembled monolayer films, Nucleic Acids Research 24 (1996), 3031-3039.

[15] E.M. Elnifro, A.M. Ashshi, R.J. Cooper and P.E. Klapper, Multiplex PCR: Optimization and application in diagnostic virology, Clinical Microbiology Reviews 13 (2000), 559-570.

[16] E. Costa, M. Rodriguez-Dominguez, M.A. Clari, E. Gimenez, J.C. Galan and D. Navarro, Comparison of the performance of 2 commercial multiplex PCR platforms for detection of respiratory viruses in upper and lower tract respiratory specimens, Diagnostic Microbiology and Infectious Disease 82 (2015), 40-43.

[17] K.L. Prime and G.M. Whitesides, Adsorption of proteins onto surfaces containing end-attached oligo (ethylene oxide) A model system using self-assembled monolayers, Journal of the American Chemical Society 115 (1993), 10714- 
10721.

[18] H. Ma, J.A. He, X. Liu, J. Gan, G. Jin and J. Zhou, Surface initiated polymerization from substrates of low initiator density and its applications in biosensors, ACS applied materials \& interfaces 2 (2010), 3223-3230.

[19] J.A. He, F. Zhao, C. Wu, J. Yao, L. Shi, C. Liu, C. Zhao, Y. Xu, X.A. Wang and D. Gu, Development of a smart dynamic surface chemistry for surface plasmon resonance-based sensors for the detection of DNA molecules, Journal of Materials Chemistry B 1 (2013), 5398-5402. 